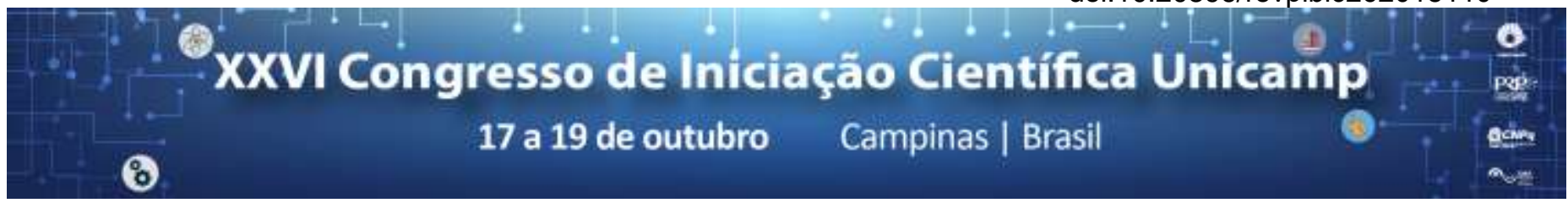

\title{
Nanostructured Lipid Carriers with ultra purified lipids for sustained release of intravenous lidocaine
}

\section{Ana Carolina Martins Pelegrine*, Viviane Lucia Beraldo de Araújo, Laura de Oliveira Nascimento.}

\begin{abstract}
Lidocaine (LDC) is a local anesthetic that can be used for post-surgical or cancer related pain, via intravenous infusion or bolus. An alternative to maintain drug plasma levels are the sustained release carriers, whereas nanostructured lipid carriers (NLCs) stand out; they have biocompatibility, low toxicity, reproducibility and high efficiency in the transport of hydrophobic drugs. This work aims to study the solubility parameter of the LDC pre formulation in several ultra-purified lipids, compatible with the endovenous route, and to formulate NLCs based on these lipids for LDC encapsulation.
\end{abstract}

Key words: Lidocaine, intravenous, lipids nanocarriers.

\section{Introduction}

There are several classes of medications used to combat chronic pain or pain related to post-operative situations. Among them is lidocaine (LDC), which is a local anesthetic and can be used intravenously by infusion or various bolus because of its short plasma half-life; these conditions require additional patient controls, equipment and/or higher doses of the drug. This problem can be solved by administering LDC in a sustained-release pharmaceutical form. The drug delivery system (DDS) encompass some classes, including nanostructured lipid carriers (NLCs), and have the advantages of being biocompatible, biodegradable and have flexibility in relation to the nature of the encapsulated drug. These NLCs are of interest to the pharmaceutical industry as they can be scalable and can be formulated with inexpensive excipients.

\section{Results and Discussion}

The apparent solubility of LDC was determined in liquid and liquefied solid lipids, with increasing LDC addition, followed by stirring, until it is no longer soluble in the medium visually (solids). The results obtained are shown in Table 1, as well as the lipids used.

Table 1. Lidocaine solubility in different lipids

\begin{tabular}{|c|c|c|c|c|c|c|}
\hline $\mathrm{CP}$ & $\mathrm{CA}$ & $\mathrm{OG}$ & $\mathrm{OR}$ & $\mathrm{OA}$ & $\mathrm{OM}$ \\
\hline 4 & 7 & 160 & 260 & 120 & 160 \\
$\mathrm{mg} / \mathrm{mg}$ & $\mathrm{mg} / \mathrm{mg}$ & $\mathrm{mg} / \mathrm{ml}$ & $\mathrm{mg} / \mathrm{ml}$ & $\mathrm{mg} / \mathrm{ml}$ & $\mathrm{mg} / \mathrm{ml}$
\end{tabular}

Legend: Cetyl palmitate wax (CP), beeswax (CA), sesame oil $(\mathrm{OG})$, castor oil (OR), cottonseed oil (OA) and corn oil (OM).

LDC solubilized in all lipids tested, but with best results in castor oil, cetyl palmitate and beeswax. Then, NLCs were prepared by the hot emulsification and sonication method and characterized by determination of the mean hydrodynamic diameter of the particles (size) by dynamic light scattering (DLS), and the zeta potential was determined by electrophoretic mobility. Determination of the encapsulation efficiency (EE) was made by the indirect method, quantifying the non-encapsulated drug by HPLC. The results are shown in Table 2.

\begin{tabular}{|c|c|c|c|}
\hline \multicolumn{4}{|c|}{ Table 2. Characterization of NLCs } \\
\begin{tabular}{|c|c|c|} 
Z-AVERAGE \\
(nm)
\end{tabular} & PDI & $\begin{array}{c}\zeta \text { Potential } \\
(\mathrm{mV})\end{array}$ & EE (\%) \\
\hline $371 \pm 5$ & $0,31 \pm 0,04$ & $-46,6 \pm 4,3$ & $68,6 \pm 1,14$ \\
\hline
\end{tabular}

The parameters of the characterization were adequate according to the literature and with satisfactory encapsulation efficiency.

The critical temperatures of the formulations, determined by lyophilization microscopy, is $-16.1^{\circ} \mathrm{C}$. The dried, collapsed and frozen layers are identified as 1,2 and 3 respectively in the Fig. 1.

Fig. 1. Determination of NLCs critical temperatures by lyophilization microscopy. Collapsed sample at $-6.9^{\circ} \mathrm{C}$.

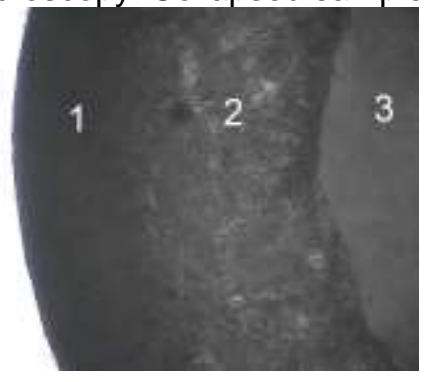

The critical temperature was adequate for the sample to be lyophilized without further excipients.

\section{Conclusions}

Overall, ultrapurified lipids presented a good diluent for LDC and NLCs showed good parameters to carry the anesthetic.

\section{Acknowledgement}

CNPQ for the scholarship.

1.Kingsley, J. D. et al. J. Soc. NeuroImmune Pharmacol. 1, 340-350 (2006). 2.De Araújo, D. R. et al. Expert Opin. Drug Deliv. 10, 1551-1563 (2013). 